\title{
Influence of High-speed Elevator Operation Parameters on Longitudinal Vibration of Time- varying Hoisting System
}

\author{
Shuohua Zhang \\ School of mechanical and electrical engineering \\ Shandong Jianzhu University \\ Jinan,China \\ 884799801@qq.com \\ Shunxin Cao \\ School of mechanical and electrical engineering \\ Shandong Jianzhu University \\ Jinan,China \\ 1140286117@qq.com
}

\begin{abstract}
Based on the finite deformation theory and Hamliton principle, considering the time-varying characteristics of the steel wire rope, the longitudinal vibration time - varying model of high - speed elevator hoisting system is established , and the longitudinal vibration dynamic equation of hoisting system is obtained. Though the precise integration method, the motion state curve and elevator car's longitudinal vibration acceleration curve of the high-speed elevator under different operating parameters are obtained. The results show that within a certain range, the upstream acceleration has a significant effect on the longitudinal vibration of the hoisting system; With the increase of the upstream acceleration, the longitudinal vibration displacement of the elevator car increases obviously; With the increase of the upstream acceleration, the acceleration of the longitudinal vibration of the car is firstly obviously increased and then Increasing trend tends to be slow.
\end{abstract}

Keywords-high-speed elevator; time-varying; longitudinal vibration; operation parameters.

\section{INTRODUCTION}

The rise of high-rise and super high-rise buildings makes the popularization of high-speed elevators become the general trend. The hoisting speed of high-speed elevator (especially in the acceleration and deceleration stages) will have an important influence on the longitudinal vibration of the elevator. For the dynamic characteristics of high-speed elevators, a large number of scholars have conducted relevant research, and achieved many useful results [1-4]. These studies are often used to set the operating parameters of the elevator (this article will be high-speed elevator upstream stage's acceleration and uniform speed running speed are collectively referred to as the operating parameters) into a fixed value, on this basis, the influence of elevator model and various uncertain structural parameters on the longitudinal vibration of elevator is studied [5, 6], and the system law between elevator operating parameters and elevator longitudinal vibration is lack.

1 The Natural Science Foundation of Shandong Province, Project number: ZR2017MEE049

2 Introduce urgently needed talents project for the western economic uplift belt and the key areas of poverty alleviation and development in Shandong Province

\author{
Ruijun Zhang \\ School of mechanical and electrical engineering \\ Shandong Jianzhu University \\ Jinan,China \\ zhangruijun@sdjzu.edu.cn \\ Qing Zhang \\ School of mechanical and electrical engineering \\ Shandong Jianzhu University \\ Jinan,China \\ zhangqing@sdjzu.edu.cn
}

In view of the above shortcomings, this paper firstly based on considering the time-varying characteristics of wire rope, the time-varying model of longitudinal vibration of high-speed elevator hoisting system were established, and then uses the continuous medium finite deformation theory and Hamliton principle $[5,6]$ to obtain the longitudinal vibration dynamic equation of hoisting system of high-speed traction elevator. Though the precise integration method, the motion state curve and elevator car's longitudinal vibration acceleration curve of the high-speed elevator under different operating parameters are obtained. Through comparison and analysis, the law between elevator operating parameters and elevator longitudinal vibration is obtained, which provides reference for the design of high-speed elevator operation parameters.

\section{High - SPEEd EleVAtor Hoisting System} LONGITUDINAL VIBRATION TIME - VARYING MODEL

The linear density of the hoisting rope is $\rho$, the cross sectional area is $A$, the elastic modulus is $E$, the length of the hoisting rope is $l(t)$, and the vertical downward direction is the positive direction of the $x$ axis. In order to facilitate the analysis of high-speed elevator operating parameters, the modeling and solving of this paper is based on the following three assumptions $[8,9]$ :

(1) The linear density $\rho$, cross-sectional area $A$ and elastic model $E$ of the high-speed elevator hoisting rope are always kept constant during operation.

(2) This paper mainly studies the longitudinal vibration of the elevator system, so it does not consider the influence of the lateral excitation of the hoisting rope, and the elastic deformation produced by the longitudinal vibration of the hoisting rope is much smaller than the length of the hoisting rope.

(3) The influence of well flow, external excitation and the friction of the steering system is not considered. 
The vibration displacement at the string $x(t)$ is $y(x(t), t)$, $v(t)$ is the speed of the high-speed traction elevator, and the longitudinal vibration time-varying model of the high-speed elevator hoisting system is set up as shown in Fig. 2.

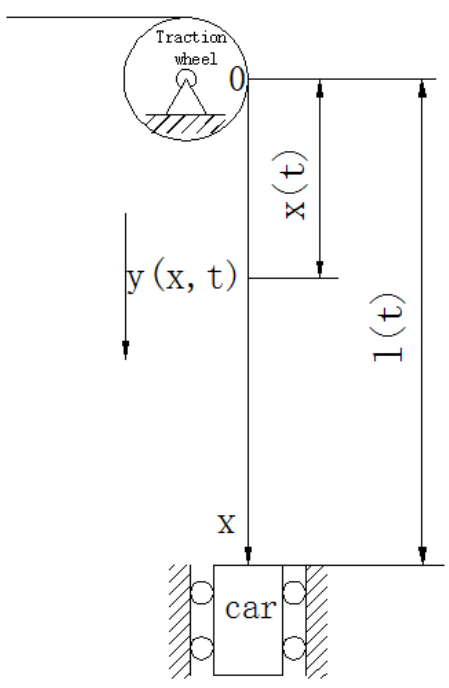

Fig. 1. Hoisting system dynamics model.

Using the finite deformation theory of continuous medium, the displacement vector and velocity vector in the $x$ axis of $x(t)$ are as follows:

$$
\begin{gathered}
r=[x(t)+y(x(t), t)] j \\
V=\left[v(t)+y_{t}(x(t), t)\right] j
\end{gathered}
$$

Where $j$ is the unit vector of $x$ along the axis direction, and $y_{t}(x(t), t)$ is the partial derivative of $y(x(t), t)$ versus $t$, the following were used $y, y_{t}$ to represent $y(x(t), t)$, $y_{t}(x(t), t)$.

Similarly, it can be seen that the displacement vector and velocity vector of the elevator car in the axial direction are as follows,

$$
\begin{gathered}
r_{c}=[l(t)+y] j \\
V_{c}=\left[v(t)+y_{t}\right] j
\end{gathered}
$$

The system kinetic energy can be expressed as:

$$
E_{\mathrm{k}}=\left.\frac{1}{2} m V^{2}\right|_{x=l(t)}+\frac{1}{2} \rho_{1} \int_{0}^{l(t)} V^{2} d s
$$

The system elastic potential energy can be expressed as:

$$
E_{\mathrm{s}}=\int_{0}^{l(t)}\left(P y_{x}+\frac{1}{2} E A y_{x}^{2}\right) d s
$$

$y_{x}(x(t), t)$ is the partial derivative of $x$, the following $y_{x}$ is used to denote $y_{x}(x(t), t)$.

$P$ is the tensioning force of the hoisting rope in the static equilibrium state

$$
P=[\mathrm{m}+\rho(l(t)-x)] g+F_{f}
$$

The gravitational potential energy of the system is expressed as:

$$
E_{\mathrm{g}}=-\int_{0}^{l(t)} \rho_{1} g d t-\left.m g y\right|_{x=l(t)}
$$

According to Hamliton principle:

$$
I=\int_{t_{1}}^{t_{2}}\left[\delta E_{k}-\delta E_{s}-\delta E_{g}\right] d t=0
$$

And using the temporal boundary conditions and geometric boundary conditions:

$$
\delta y(0, t)=\delta y\left(x, t_{1}\right)=\delta y\left(x, t_{2}\right)=0
$$

The longitudinal vibration dynamic equation of high-speed elevator hoisting system is obtained:

$$
\begin{aligned}
& \rho\left(y_{t t}+a\right)-P_{x}-\rho g-E A y_{x x}=0,0<x<l(t) \\
& m\left(a+y_{t t}\right)+\rho v\left(v+y_{t}\right)+E A y_{x}+P-m g \\
& =0, x=l(t)
\end{aligned}
$$

Formula (12) is the boundary condition of the chord at $x=l(t)$

The longitudinal vibration dynamic equation of the abovementioned high-speed elevator hoisting system are normalized discrete, and the original partial differential control equations are discretized into the following equations:

$$
M \ddot{q}_{j}+C \dot{q}_{j}+K q_{j}=F
$$

In the formula, $q_{j}=\left[q_{1}(t), q_{2}(t), \cdots, q_{n}(t)\right]$ is the generalized coordinate vector,

$$
M=\rho \delta_{i j}+\frac{m}{l} \varphi_{i}(1) \varphi_{j}(1)
$$


$C=-\frac{2 \rho v}{l} \int_{0}^{1} \xi \varphi_{i}^{\prime} \varphi_{j} d \xi+\frac{\rho v}{l} \varphi_{i}(1) \varphi_{j}(1)$

$K=\frac{m v^{2}}{l^{3}} \varphi_{i}^{\prime \prime}(1) \varphi_{j}(1)-\frac{\rho a}{l} \int_{0}^{1} \xi \varphi_{i}^{\prime} \varphi_{j} d \xi-\frac{\rho v^{2}}{l^{2}} \int_{0}^{1} \xi^{2} \varphi_{i}^{\prime} \varphi_{j}^{\prime} d \xi-\frac{E A}{l^{2}} \int_{0}^{1} \varphi_{i}^{\prime \prime} \varphi_{j} d \xi$

$$
F=-\rho a \int_{0}^{1} \varphi_{j} d \xi-\frac{m a}{l} \varphi_{j}(1)-\frac{\rho v^{2}}{l} \varphi_{j}(1)
$$

\section{SETTING AND CALCULATION OF ELEVATOR OPERATING PARAMETERS}

In order to investigate the influence of the upstream operating parameters of the high-speed elevator on the longitudinal vibration of the time-varying hoisting system, the four accelerating accelerations were selected as the accelerating speed of $0.5 \mathrm{~m} / \mathrm{s}^{2}, 0.75 \mathrm{~m} / \mathrm{s}^{2}, 1 \mathrm{~m} / \mathrm{s}^{2}$ and $1.25 \mathrm{~m} / \mathrm{s}^{2}$, in ensuring the rated hoisting speed is constant under the condition that the speed of the elevator car under different acceleration curve and displacement curve.
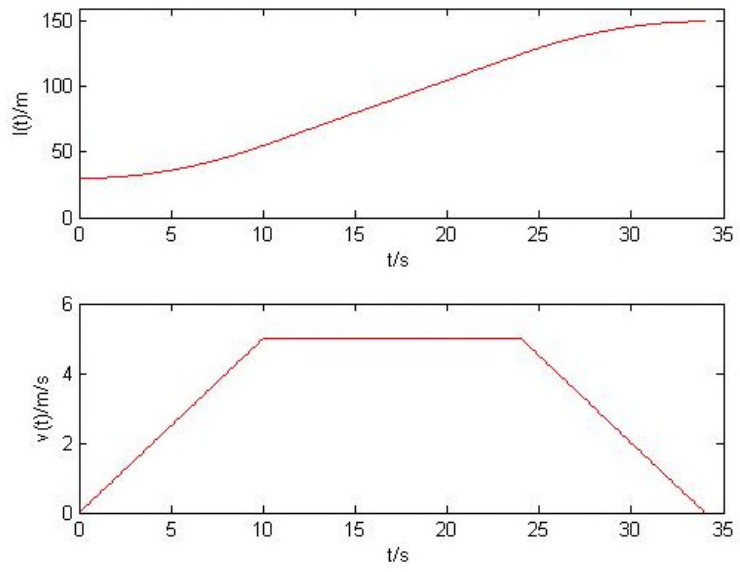

a) Lift speed and displacement of elevator the accelerating speed of $0.5 \mathrm{~m} / \mathrm{s}^{2}$
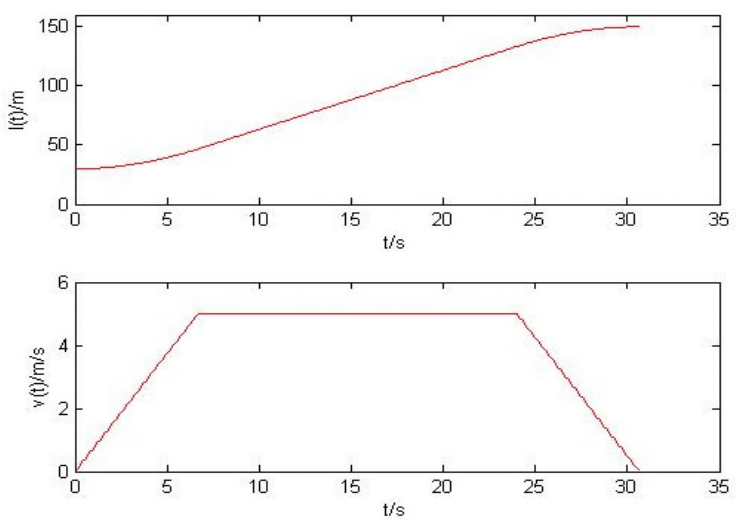

b) Lift speed and displacement of elevator the accelerating speed of $0.75 \mathrm{~m} / \mathrm{s}^{2}$
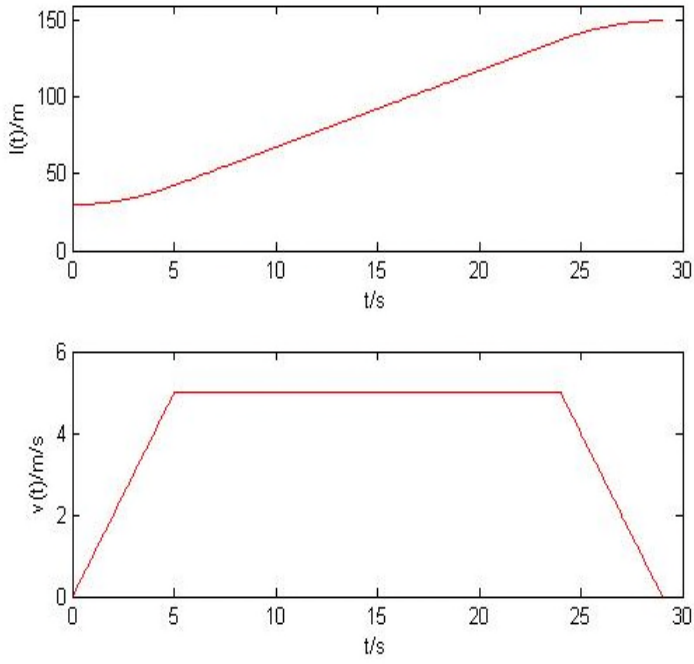

c) Lift speed and displacement of elevator the accelerating speed of $1 \mathrm{~m} / \mathrm{s}^{2}$
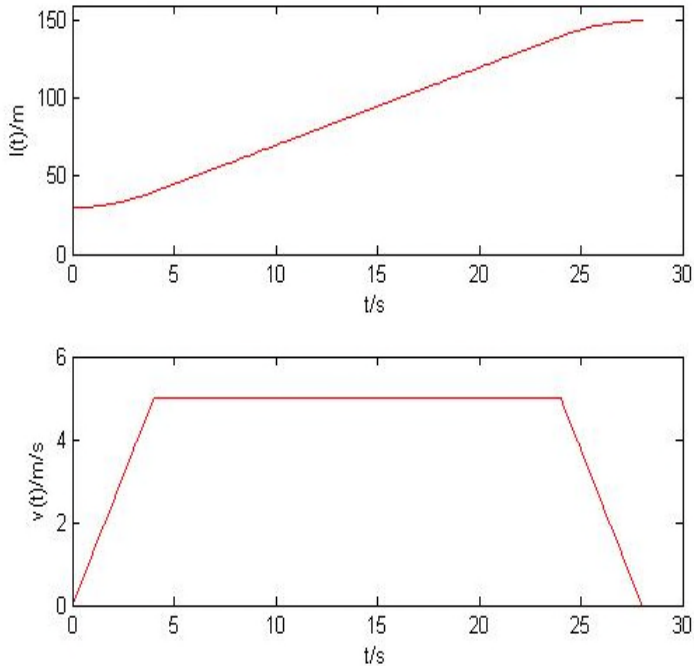

d) Lift speed and displacement of elevator the accelerating speed of $1.25 \mathrm{~m} / \mathrm{s}^{2}$

Fig. 2. Elevator operating parameters under different accelerations.

\section{CASE Simulation AND Result Analysis}

This paper takes a $5 \mathrm{~m} / \mathrm{s}$ high-speed elevator of Fuji Elevator Co. Ltd production as the research object, the elastic modulus of the hoisting rope $\mathrm{E}=13 \times 10^{10} \mathrm{~N} / \mathrm{m}^{2}$, sectional area $\mathrm{A}=89.344 \times 10^{6} \mathrm{~m}^{2}$, hoisting rope line density $\mathrm{B}=0.87 \mathrm{~kg} / \mathrm{m}$, single hoisting rope to withstand the quality of $400 \mathrm{~kg}$. The operation parameters set in Section 3 are taken into the timevarying motion equations established in Section 2, and the numerical integration is carried out by means of fine integral method. The simulation results are shown in Fig. 3: 

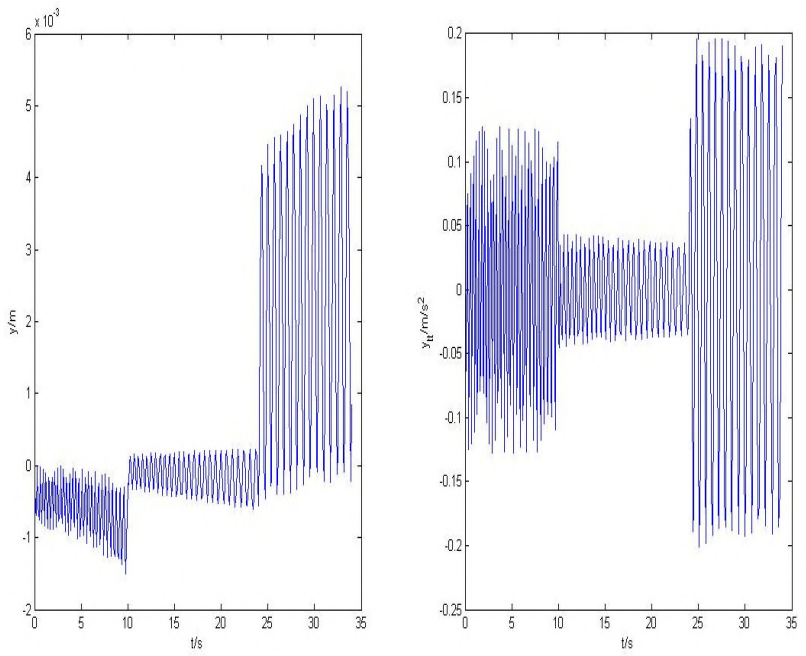

a) Longitudinal vibration curve of car under upstream acceleration $\mathrm{a}=0.5 \mathrm{~m} / \mathrm{s}^{2}$
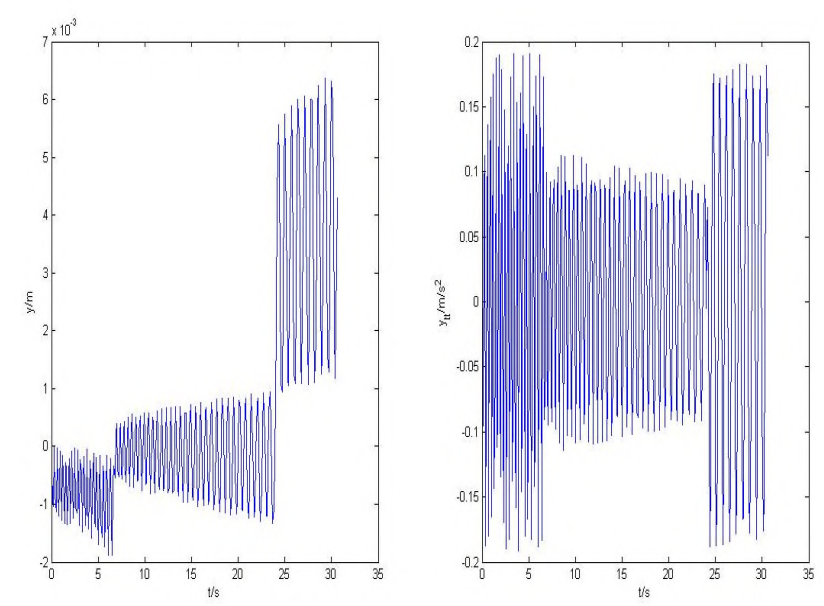

b) Longitudinal vibration curve of car under upstream acceleration $\mathrm{a}=0.75 \mathrm{~m} / \mathrm{s}^{2}$
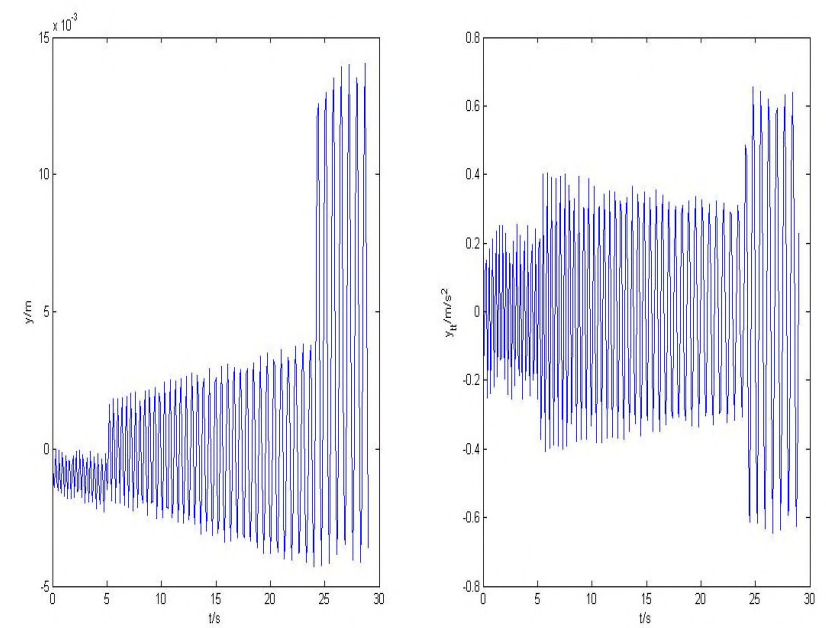

c) Longitudinal vibration curve of car under upstream acceleration $\mathrm{a}=1 \mathrm{~m} / \mathrm{s}^{2}$


d) Longitudinal vibration curve of car under upstream acceleration

$$
\mathrm{a}=1.25 \mathrm{~m} / \mathrm{s}^{2}
$$

Fig. 3 Longitudinal vibration curve of car under different upstream accelerations

It can be seen from Fig. 3, the upstream acceleration changes from $0.5 \mathrm{~m} / \mathrm{s}^{2}$ to $0.75 \mathrm{~m} / \mathrm{s}^{2}$, from $0.75 \mathrm{~m} / \mathrm{s}^{2}$ to $1 \mathrm{~m} / \mathrm{s}^{2}$, time-varying hoisting system longitudinal vibration displacement and acceleration increased significantly, the upstream acceleration changes from $1 \mathrm{~m} / \mathrm{s}^{2}$ to $1.25 \mathrm{~m} / \mathrm{s}^{2}$, the longitudinal vibration displacement of the time-varying hoisting system increases obviously, while the vibration acceleration does not change significantly.

\section{CONCLUSION}

In this paper, a time-varying model of longitudinal vibration of high-speed elevator hoisting system is established, and the law between elevator operating parameters and longitudinal vibration of elevator is explored by using this model.

Within a certain range, the upstream acceleration has a significant effect on the longitudinal vibration of the hoisting system.

The upstream acceleration increased from $0.5 \mathrm{~m} / \mathrm{s}^{2}$ to $1.25 \mathrm{~m} / \mathrm{s}^{2}$, and the longitudinal vibration displacement of the elevator car increased obviously.

The upstream acceleration is increased from $0.5 \mathrm{~m} / \mathrm{s}^{2}$ to $1.25 \mathrm{~m} / \mathrm{s}^{2}$, firstly, the acceleration of the longitudinal vibration of the car is obviously increased and then increasing trend tends to be slow.

The conclusions of this paper have certain guiding significance for the design and operation parameters setting of high-speed elevator hoisting system.

\section{REFERENCES}

[1] D. Mei, X. Du and Z. Chen, "Optimization of dynamic parameters fora traction-type passenger elevator using a dynamic byte coding genetic 
algorithm”. Proc. Inst. Mech. Eng. Part C: J. Mech. Eng. Sci. vol. 223, pp. 595-605, 2009.

[2] W.Z. Feng, S.Q. Cao. "Frequency Analysis and Robust Design of Vertical Vibration of Elevator System". Vibr. Test. Diagn. vol. 34 , no. 4, pp. 630-635, 2016.

[3] N. Chang, W. He and Z.H. Li. "Study on vibration damping of vertical vibration of elevator system". Noise Vibr. Contr. vol. 37, no. 2, pp. 117 137, 2017.

[4] H. Wu, W.H. Ye, Y. Shen and Z.R. Tang. "Modeling and Experimental Analysis of Vertical Vibration of High Speed Elevator". Mach. Manuf. vol. 51 , no. 584, pp. 19-22, 2013.

[5] L.M. Wu, Y.Y. Gong and X.F. Li. "Analysis of dynamic characteristics of vertical vibration of traction elevator mechanical system". Mach. Des. Manuf. vol. 10, pp. 16-18, 2007.
[6] W.Z. Feng, S.Q. Cao, F. Zhao, C. Hu and W.B. Liu. "Study on Sensitivity of Resonant Failure in Elevator System". J. Vibr Shock. vol. 34, no. 1, pp. 165-170, 2015..

[7] H. Wu, Y.B. Liu. "Dynamic Performance Analysis and Optimization of High-Speed Elevator based on Virtual Prototype Technology". J. Graph. vol. 34, no. 6, pp. 98-105, 2013.

[8] X.Q. Du, D.Q. Mei, Z.C. Chen. "Time-dependent Model and Analysis of Horizontal Vibration Response of High-speed Traction Elevator". J. Zhejiang Univ. vol. 43 , no. 1, pp. 148-152, 2009.

[9] J.H. Bao, C.M. Zhu. "Research on dynamic modeling and vibration control method of high-speed elevator lifting system". Shanghai Jiaotong University, 2014. 